\title{
Article \\ Ultrasound Features across Subtypes of Juvenile Idiopathic Arthritis
}

\author{
Doaa Mosad Mosa ${ }^{1, *(D)}$, Ashraf M. Abdelrahman ${ }^{2}$ and Amany S. El-Bahnasawy ${ }^{1}$ \\ 1 Rheumatology \& Rehabilitation Department, Mansoura University Hospitals, \\ Mansoura City 35516, Egypt; D_amy75@yahoo.com \\ 2 Diagnostic Radiology, Mansoura University Children's Hospital, Mansoura University, \\ Mansoura City 35516, Egypt; ashrafmuhamed2@gmail.com \\ * Correspondence: doaamosad@mans.edu.eg
}

check for updates

Citation: Mosa, D.M.; Abdelrahman, A.M.; El-Bahnasawy, A.S. Ultrasound Features across Subtypes of Juvenile Idiopathic Arthritis. Rheumato 2022, 2, 2-14. https://doi.org/10.3390/ rheumato2010002

Academic Editor: Bruce

M. Rothschild

Received: 13 December 2021

Accepted: 27 January 2022

Published: 29 January 2022

Publisher's Note: MDPI stays neutral with regard to jurisdictional claims in published maps and institutional affiliations.

Copyright: () 2022 by the authors Licensee MDPI, Basel, Switzerland. This article is an open access article distributed under the terms and conditions of the Creative Commons Attribution (CC BY) license (https:// creativecommons.org/licenses/by/ $4.0 /)$.

\begin{abstract}
Objective: The aim of this study was to evaluate musculoskeletal ultrasound (MSUS) features across categories of juvenile idiopathic arthritis (JIA). Methods: In this cross-sectional study, all patients were subjected to full history taking, clinical examination including disease assessment parameters and laboratory investigations. In addition, all children were examined by both grayscale (GS) and power Doppler (PD) MSUS images. Results: By MSUS, the number of joints with synovial effusion was 697 of a total 2400 examined joints (29\%) and joints with synovial thickening counted $673(28 \%)$. The number of joints with positive PD signals was 446 (18.6\%). There was a significant difference among JIA subtypes as regards different MSUS features. Moreover, there was a discrepancy regarding synovial effusion $(p=0.018)$, hypertrophy scores $(p=0.013)$, and the total US severity score $(p=0.026)$. This divergence was attributed to the significant difference between systemic juvenile idiopathic arthritis (SJIA) and other categories. The sensitivity, specificity, positive predictive value (PPV), negative predictive value (NPV), and accuracy of MSUS in JIA and its subtypes were calculated. Conclusion: MSUS is a highly sensitive method for detecting synovitis, tenosynovitis, and erosive bone disease, and it helps to make proper therapeutic decisions. There was a significant difference among JIA subtypes regarding MSUS features.
\end{abstract}

Keywords: juvenile idiopathic arthritis; musculoskeletal ultrasound; disease assessment; synovitis

\section{Introduction}

Juvenile idiopathic arthritis (JIA) is a chronic rheumatic disease characterized by synovitis of peripheral joints persisting more than six weeks. It affects children before the age of sixteen, and it is the most common pediatric rheumatic disease [1]. JIA is diagnosed after exclusion of other causes as infection, malignancy, and other rheumatic diseases. According to the International League of Association for Rheumatology (ILAR), it comprises seven heterogeneous subsets with unique clinical patterns of each type [2].

Identifying patients with disease activity and predicting disease outcomes form the basis of optimal clinical management. The heterogeneity of clinical manifestations of JIA and the absence of reliable markers that predict disease progression have accentuated the quest for newer measures. These tools will help in better patient classification and disease monitoring [3].

Compared to clinical examination and conventional radiology, musculoskeletal ultrasound (MSUS) is a more sensitive method for detecting synovitis, tenosynovitis, and erosive bone disease [4]. In JIA, MSUS is helpful in the detection of subclinical synovitis, early diagnosis, patient classification, disease activity monitoring, determining disease remission, and guiding intra-articular injections [5].

MSUS is a suitable imaging modality for children as it requires neither sedation nor general anesthesia and no ionizing radiation, is easily repeated, compares between joints, 
and allows dynamic study and multisite assessment in the same session [6]. This study aimed to evaluate MSUS features among different categories of JIA.

\section{Patients and Method}

This cross-sectional study was conducted on a group of children attending the rheumatology clinic at Mansoura University children's hospital (MUCH). Patients were recruited during their routine visits from August 2017 to October 2018. For ethical consideration, the study was conducted after the approval of institutional research board (IRB) of the Faculty of Medicine, Mansoura University, Egypt (approval number: M 1900483).

\subsection{Inclusion Criteria}

1-Patients who fulfilled the classification criteria of JIA according to ILAR.

2-Patients whose parents signed a consent to be included in the study.

\subsection{Exclusion Criteria}

Children with septic arthritis, hemarthrosis, and arthritis related to other causes such as malignancy, trauma, and connective tissue disorders were excluded.

\subsection{Methods}

All patients were interrogated for full medical history, clinical examination, MSUS examination, and laboratory investigations. Appropriate data about the patients were retrieved from the medical records.

\subsubsection{Disease Assessment}

Assessment of Disease Activity

Juvenile arthritis disease activity score-10 (JADAS-10): this includes four components [7]. The disease activity was defined as $>2$ in oligoarticular subtype and $>3.8$ in polyarticular and systemic juvenile idiopathic arthritis (SJIA) subtypes.

Juvenile spondyloarhritis disease activity (JSPADA) score was used for enthesitisrelated arthritis cases (ERA). This index includes 8 items that are equally weighted and based mainly on physician assessment [8]. The total score is gained by the simple numeric addition of all components, and it ranges from 0 to 8 .

\section{Assessment of Disease Damage}

The amount of disease damage articular and extra-articular was measured by Juvenile Arthritis Damage Index (JADI) [9].

\section{Assessment of Function}

Child health assessment questionnaire (C-HAQ) that describes the child usual activities in 8 domains over the past week (dressing, grooming, arising, eating, walking with or with assistive devices, hygiene, reach, and grip) [10]. Each question was scored from 0 to 3 $(0=$ no difficulty $1=$ some difficulty $2=$ much difficulty $3=$ unable to do $)$, and the score of these 8 functions was averaged to calculate the disability index.

\subsubsection{Laboratory Investigations Included}

Complete blood count (CBC), erythrocyte sedimentation rate (ESR), C -reactive protein $(C R P)$, liver function tests, serum creatinine, and rheumatoid factor $(\mathrm{RF})$.

\subsubsection{MSUS}

It was performed by an experienced radiologist who was blinded to the diagnosis of each child. A PHILPS IU22 apparatus was used with a linear transducer at a frequency of 12 megahertz (MH). All children were examined by both grayscale (GS) and power Doppler (PD) images. The PD settings were the following: the pulse repetition frequency (PRF) ranged from 600 to 800 hertz (Hz) depending on examined joint, there was a low wall 
filter, and the PD gain was set at the highest level at which the PD signal did not appear under the bony cortex. The same 40 joints assessed clinically for each patient (right and left elbows, wrists, metacarpophalangeal (MCP) joints, proximal interphalangeal (PIP) joints, knees, ankles, subtalar, and metatarsophalangeal joints) were scanned for:

1. Synovial thickening

2. Synovial effusion

3. Tenosynovitis

4. Bone erosions

5. Cartilage thickness in $\mathrm{mm}$

6. PD signals

All ultrasound (US) findings were interpreted on GS images using both longitudinal and transverse planes. The joints were examined according to the recommendations for standardized scanning by the outcome measures in rheumatology clinical trials (OMERACT) pediatric ultrasound group [11]. The US examination techniques as well as the definitions of US features were based on the European league against rheumatism (EULAR) and the OMERACT guidelines, which graduated both GS and PD into 3 grades based on their extension $[12,13]$.

The US score for synovial thickening, synovial effusion, and PD (active synovitis) was calculated as the sum of severity rating obtained from each joint (each ranging from 0-120). The overall US severity score was summated from the total score of synovial thickening, synovial effusion, and PD (0-360).

\section{Statistical Analysis}

All findings were statistically evaluated using Microsoft Excel and SPSS (statistical package for social science) software, version 15.0 (SPSS Inc., Chicago, IL, USA). Continuous data were expressed as mean \pm standard deviation (SD), while categorical data were expressed in number and percentage. All continuous data were tested for normal distribution before any statistical analysis. Correlation analysis was used to assess the strength of association between two quantitative variables; the correlation coefficient defined the strength and direction of the linear relationship between two variables. The diagnostic value of the active joint count and US activity score in disease activity prediction was also calculated by using the receiver operating characteristic (ROC) curve. A p-value less than 0.05 was considered statistically significant.

\section{Results}

A total 60 JIA patients were included in this study. The age of JIA patients ranged from 4 to 15 years with a mean $( \pm S D)$ of $10.7 \pm 3.5$. They included 35 females $(58.3 \%)$ and 25 males (41.7\%). Disease duration ranged from 6 months to 12 years with a median (IQR) of 3 [4.9]. Frequency distribution of different categories are displayed in (Figure 1), and their clinical data and laboratory findings are shown in Tables 1 and 2.

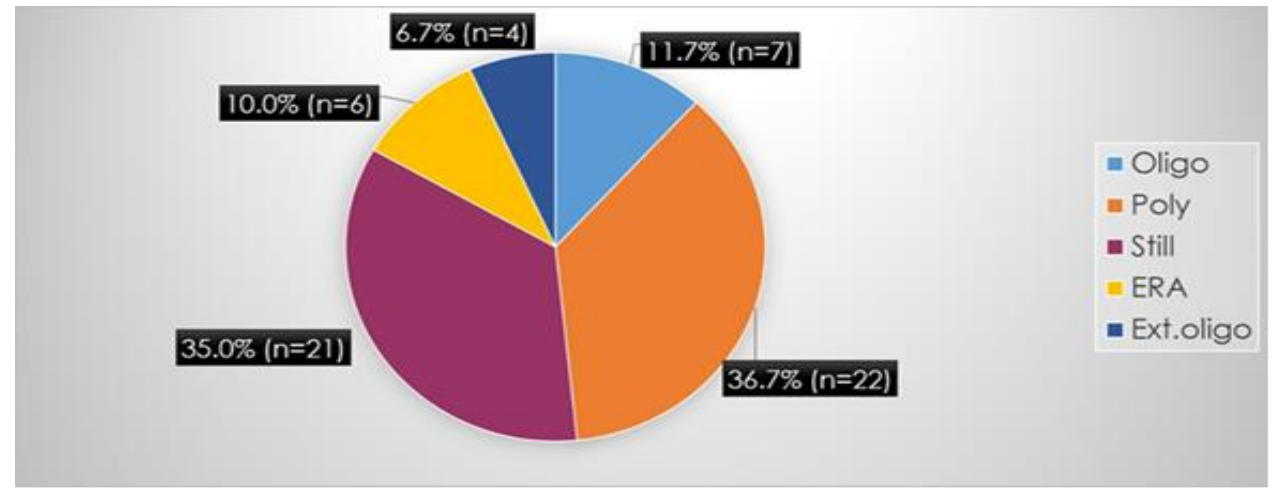

Figure 1. Prevalence of JIA subtypes. 
Table 1. Comparison of clinical data among JIA subtypes.

\begin{tabular}{|c|c|c|c|c|c|c|c|}
\hline \multicolumn{2}{|c|}{ Clinical Data } & \multicolumn{5}{|c|}{ Subtypes of JIA } & \multirow{3}{*}{$\begin{array}{c}\text { Kruskal- } \\
\text { Wallis Test, } \\
p \text { Value }\end{array}$} \\
\hline & & $\begin{array}{c}\text { Oligoarticular } \\
\quad n=7\end{array}$ & $\begin{array}{c}\text { Extended- } \\
\text { Oligo } \\
n=4\end{array}$ & $\begin{array}{c}\text { Polyarticular } \\
n=22\end{array}$ & $\begin{array}{c}\text { SJIA } \\
n=21\end{array}$ & $\begin{array}{l}\text { ERA } \\
n=6\end{array}$ & \\
\hline \multicolumn{2}{|c|}{$\begin{array}{l}\text { Total Number of } \\
\text { Examined Joints }\end{array}$} & 280 & 160 & 880 & 840 & 240 & \\
\hline \multirow{4}{*}{$\begin{array}{l}\text { Active joint } \\
\text { count }\end{array}$} & Range & $0-4$ & $0-6$ & 0-14 & $0-40$ & $2-10$ & \multirow{4}{*}{0.135} \\
\hline & Median & 2 & 3.5 & 1 & 4 & 3 & \\
\hline & (IQR) & (1) & $(5.8)$ & $(4)$ & $(10)$ & (3.5) & \\
\hline & $n$, percent & $\begin{array}{c}13 \\
(4.6 \%)\end{array}$ & $\begin{array}{c}13 \\
(8.1 \%)\end{array}$ & $\begin{array}{c}58 \\
(6.6 \%) \\
\end{array}$ & $\begin{array}{c}166 \\
(19.8 \%)\end{array}$ & $\begin{array}{c}24 \\
(10 \%)\end{array}$ & \\
\hline \multirow{4}{*}{$\begin{array}{c}\text { Tender joint } \\
\text { count }\end{array}$} & Range & $0-4$ & $1-8$ & $0-14$ & $0-40$ & $2-10$ & \multirow{4}{*}{0.135} \\
\hline & Median & 2 & 6 & 2 & 4 & 4 & \\
\hline & (IQR) & (3) & $(5.3)$ & (6) & $(12.5)$ & $(5)$ & \\
\hline & $n$, percent & $\begin{array}{c}15 \\
(5.4 \%) \\
\end{array}$ & $\begin{array}{c}21 \\
(13.1 \%)\end{array}$ & $\begin{array}{c}83 \\
(9.4 \%) \\
\end{array}$ & $\begin{array}{c}181 \\
(21.5 \%)\end{array}$ & $\begin{array}{c}28 \\
(11 \%)\end{array}$ & \\
\hline \multirow{4}{*}{$\begin{array}{l}\text { Swollen joint } \\
\text { count }\end{array}$} & Range & $0-4$ & $0-6$ & $0-26$ & $0-40$ & $2-10$ & \multirow{4}{*}{0.164} \\
\hline & Median & 2 & 3.5 & 2 & 4 & 3 & \\
\hline & (IQR) & $(1)$ & $(5.8)$ & (6) & $(9.5)$ & (3.5) & \\
\hline & $n$, percent & $\begin{array}{c}13 \\
(4.6 \%) \\
\end{array}$ & $\begin{array}{c}13 \\
(8.1 \%) \\
\end{array}$ & $\begin{array}{c}94 \\
(10.7 \%) \\
\end{array}$ & $\begin{array}{c}168 \\
(20 \%) \\
\end{array}$ & $\begin{array}{c}24 \\
(10 \%) \\
\end{array}$ & \\
\hline \multirow{4}{*}{$\begin{array}{l}\text { Limited joint } \\
\text { count }\end{array}$} & Range & $0-2$ & $0-6$ & $0-20$ & $0-40$ & $0-10$ & \multirow{4}{*}{0.320} \\
\hline & Median & 0 & 2 & 1 & 2 & 4 & \\
\hline & (IQR) & $(0)$ & $(5.5)$ & $(4)$ & $(8)$ & $(5.5)$ & \\
\hline & $n$, percent & $2(0.7 \%)$ & $10(6.3 \%)$ & $68(7.7 \%)$ & $\begin{array}{c}164 \\
(17.4 \%)\end{array}$ & $\begin{array}{c}22 \\
(9.2 \%)\end{array}$ & \\
\hline \multirow{3}{*}{ JADAS-10 } & Range & $0-11$ & $2.2-22$ & $0-38$ & $0.3-40$ & $6.5-40$ & \multirow{3}{*}{0.050 * } \\
\hline & Median & 4.5 & 12.9 & 7.9 & 21.3 & 13 & \\
\hline & (IQR) & $(4.3)$ & (19.1]) & $(7.6)$ & $(33.9)$ & $(21.3)$ & \\
\hline \multirow{3}{*}{ C-HAQ } & Range & $0-1$ & $0-2$ & $0-2.5$ & $0-3$ & $0-3$ & \multirow{3}{*}{0.035 * } \\
\hline & Median & 0 & 1 & 1 & 2 & 1.5 & \\
\hline & (IQR) & $(0)$ & $(1.75)$ & $(2)$ & $(2.5)$ & (1.5) & \\
\hline \multirow{3}{*}{ JAD-articular } & Range & $0-2$ & $0-4$ & $0-30$ & $0-40$ & $0-6$ & \multirow{3}{*}{0.722} \\
\hline & Median & 0 & 2 & 0 & 0 & 3 & \\
\hline & (IQR) & $(0)$ & $(4)$ & $(4)$ & $(5.5)$ & $(4.5)$ & \\
\hline \multirow{3}{*}{$\begin{array}{l}\text { JAD-extra } \\
\text { articular }\end{array}$} & Range & $0-2$ & $0-2$ & $0-5$ & $0-5$ & $0-1$ & \multirow{3}{*}{0.618} \\
\hline & Median & 0 & 0 & 1 & 1 & 1 & \\
\hline & (IQR) & (2) & (1.5) & (1) & (3) & $(0.25)$ & \\
\hline
\end{tabular}


Table 2. Comparison of laboratory findings among JIA subtypes.

\begin{tabular}{|c|c|c|c|c|c|c|c|}
\hline & & & & Subtypes of JIA & & & \\
\hline Labora & Data & Oligoarticular & Extended- & Polyarticular & SJIA & ERA & Kruskal- \\
\hline & & $n=7$ & $n=4$ & $n=22$ & $n=21$ & $n=6$ & $p$ Value \\
\hline ESR 1st hour & median & 30.0 & 25.5 & 26 & 60.0 & 20 & (2006 \\
\hline$(\mathrm{mm})$ & (IQR) & $(7.0)$ & $(6.2)$ & (31.25) & $(71.0)$ & (39) & 0.090 \\
\hline$C$ & median & 0.5 & 0.5 & 0.65 & 24.0 & 17.4 & 0004 \\
\hline (IU (II)/ UL) & (IQR) & (5.5) & (5.5) & (23.5) & $(40.0)$ & $(15.2)$ & 0.034 \\
\hline
\end{tabular}

SJIA: systemic juvenile idiopathic arthritis, ERA: enthesitis related arthritis, ESR: erythrocyte sedimentation rate, CRP: C -reactive protein, IQR: interquartile range. * Statistically significant $p$-values

The most frequently used drugs were methotrexate in 43 patients (71.7\%) and steroids in 34 patients $(56.7 \%)$, followed by both sulfasalazine and NSAIDS in eight cases (13.3\%). Less frequently used drugs were leflunomide, hydroxychloroquine, and cyclosporine in four $(6.7 \%)$, three $(5 \%)$, and two $(3.3 \%)$ cases, respectively. Meanwhile, biological therapy was used in eight patients (seven of them took tocilizumab (11.7\%) and one received infliximab $(1.7 \%))$. Combination therapy was prescribed in 38 patients $(63.3 \%)$.

By US examination, the number of joints with synovial effusion was 697 of total 2400 examined joints (29\%), joints with synovial thickening counted $673(28 \%)$. The number of joints with positive PD signals was 446 (18.6\%), and the number of joints with any of the previous 3 US abnormalities (US synovitis) was 730 (30.4\%). Meanwhile, tenosynovitis and erosion were detected in 38 (1.6\%), 24 (1\%) joints, respectively. Subclinical synovitis was found in 312 joints (13\%), the number of joints with low cartilage thickness was $146(0.6 \%)$, and enthesitis was detected in 28 entheses sites (4.6\%) (Figures 2-6).

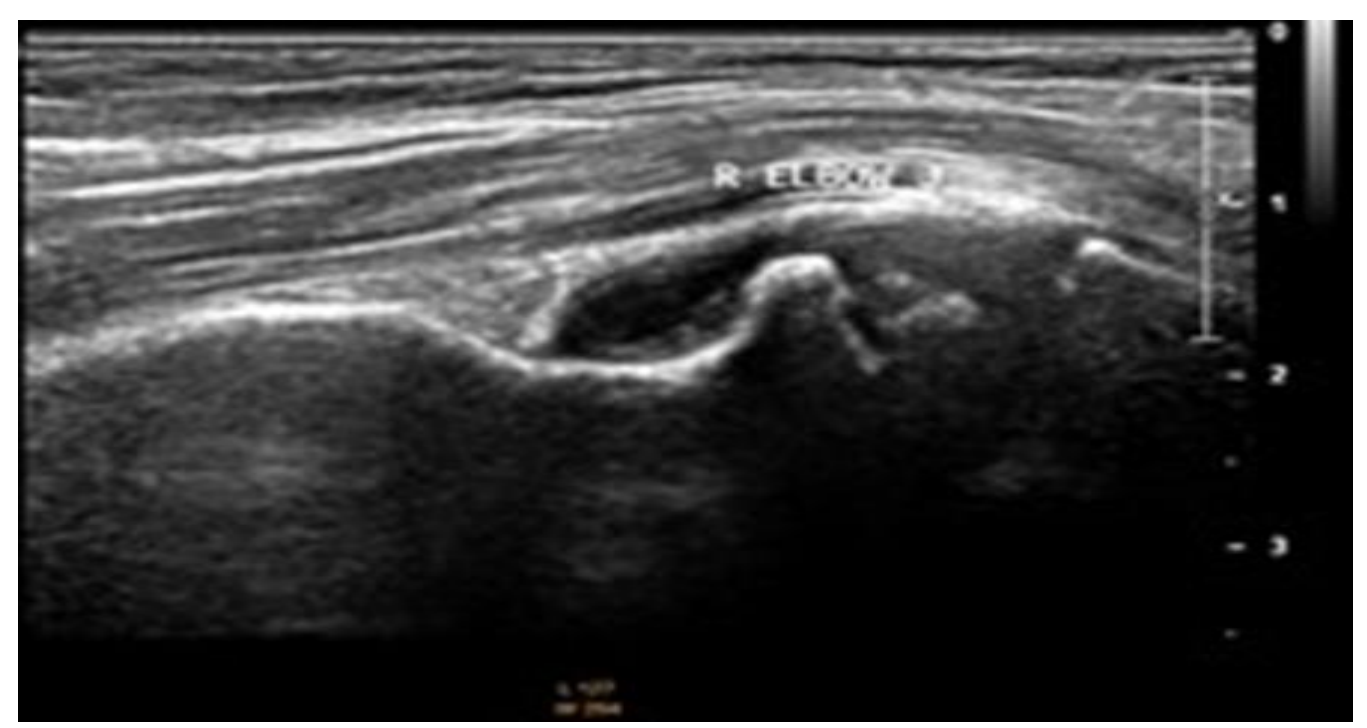

(a)

Figure 2. Cont. 


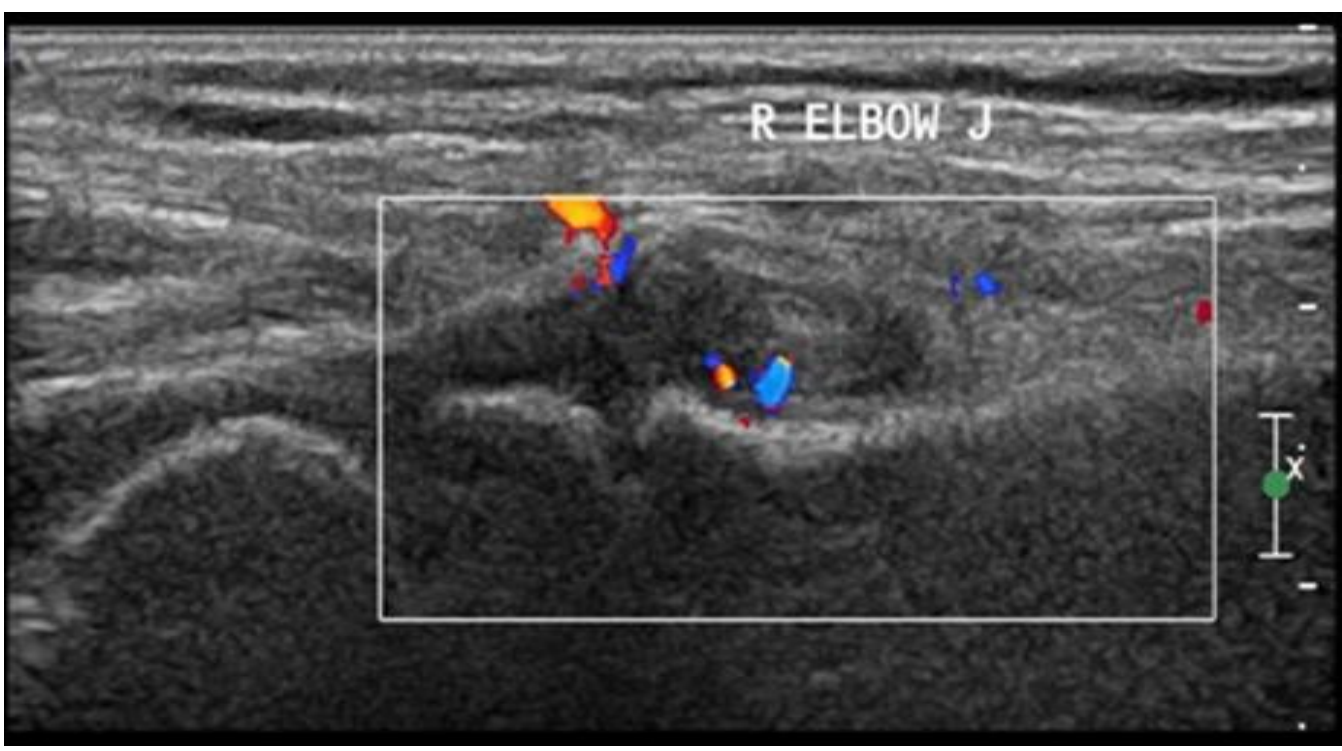

(b)

Figure 2. (a) Grade 2 synovitis of right elbow joint (longitudinal view); (b). Grade 2 PD signals of right elbow synovitis (longitudinal view).

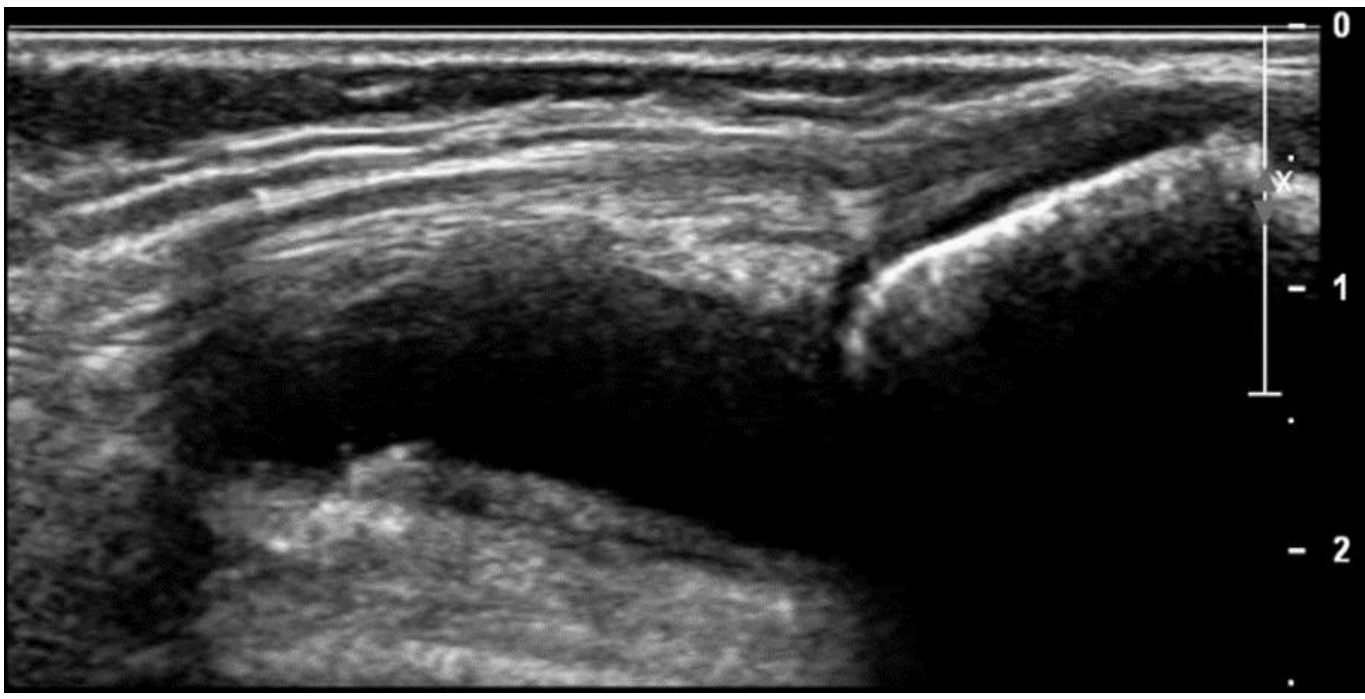

Figure 3. Grade 3 synovitis of right knee joint (longitudinal view).

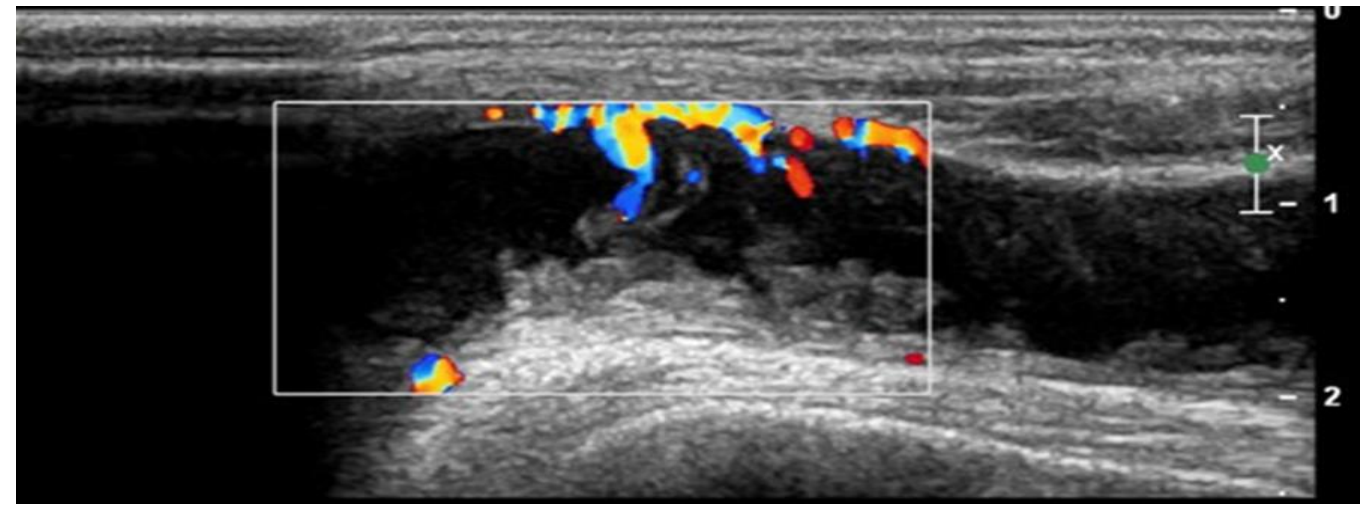

Figure 4. Grade 2 PD signals of right knee joint (transverse view). 


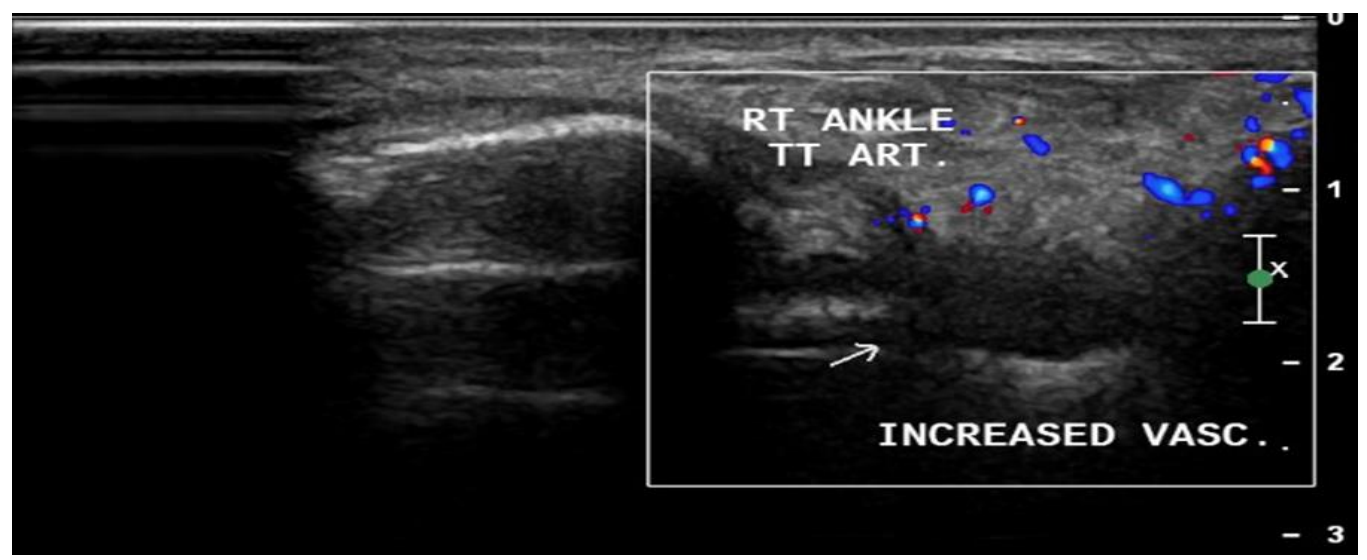

Figure 5. Grade 3 PD signals of right ankle joint (longitudinal view).

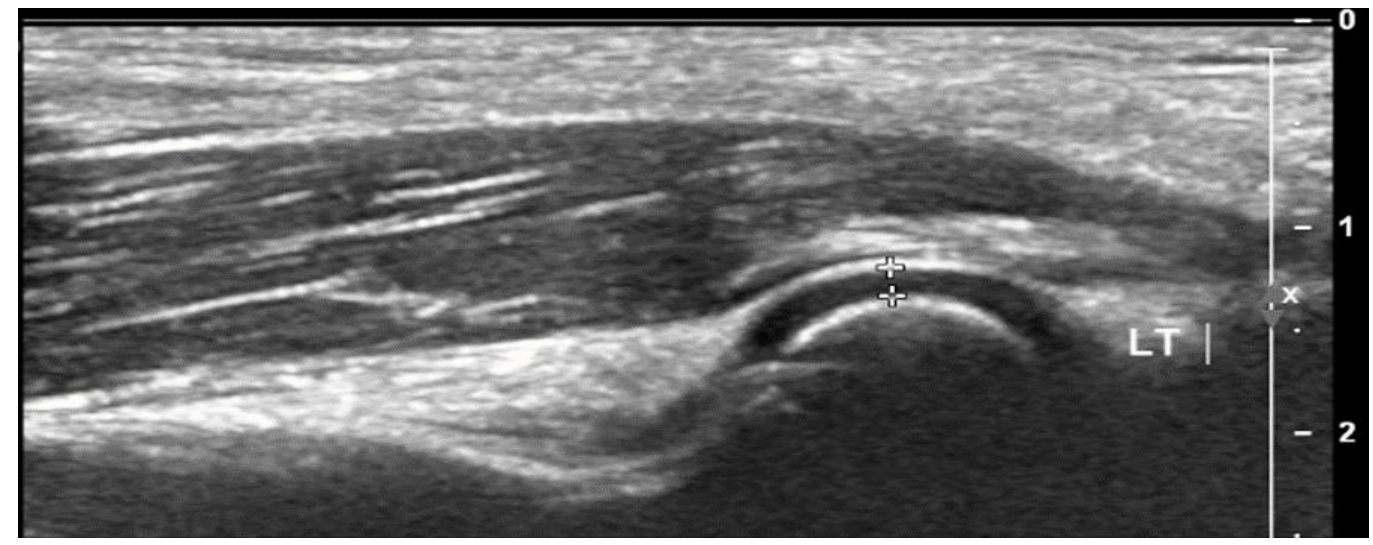

Figure 6. Measurement of cartilage thickness of humeral condyle.

The most common sites of enthesitis were the Achilles tendon followed by distal patellar tendon and plantar fascia. Moreover, enthesitis was detected in ERA and 2 cases of oligoarticular JIA. The median (IQR) of synovial effusion and thickening score was 12 [19.8], 9.5 [14], respectively. The active synovitis score had a median (IQR) of 4 [19.8], while the total US severity score had a median (IQR) of 25.5 [54.3]. The US led to classify 3 patients who had oligoarthritis on clinical examination as a polyarticular subtype (2 were RF positive and 1 was RF negative). The prevalence of US synovitis in specific joints is shown in Table 3.

Table 3. Prevalence of US synovitis in specific joints (total 730).

\begin{tabular}{ccc}
\hline & $n$ & $\%$ \\
\hline Elbow & 92 & 12.6 \\
\hline Wrist & 100 & 13.7 \\
\hline MCP & 98 & 13.4 \\
\hline PIP & 76 & 10.4 \\
\hline Knee & 110 & 15 \\
\hline Ankle & 93 & 12.7 \\
\hline Subtalar & 78 & 10.7 \\
\hline MTP & 83 & 11.4 \\
\hline n: number, MCP: metacarpophalangeal joint, PIP: proximal interphalangeal joint, MTP: metatarspalangeal
\end{tabular}

n: number, MCP: metacarpophalangeal joint, PIP: proximal interphalangeal joint, MTP: metatarsophalangeal. 
Among inflammatory markers only CRP $(p=0.034)$ showed a statistical difference between different JIA subtypes. Similarly, there was a significant difference among JIA subtypes as regards different US features as presented in Table 4 attributed to the significant difference between SJIA and other subtypes.

Table 4. Comparison of US features among JIA subtypes.

\begin{tabular}{|c|c|c|c|c|c|c|c|}
\hline \multicolumn{2}{|c|}{ US Features } & \multicolumn{5}{|c|}{ Subtypes of JIA } & \multirow{3}{*}{$\begin{array}{c}\text { Kruskal- } \\
\text { Wallis Test, } \\
p \text { Value }\end{array}$} \\
\hline & & $\begin{array}{l}\text { Oligoarticular } \\
\quad n=7\end{array}$ & $\begin{array}{l}\text { Extended- } \\
\text { Oligo } \\
n=4\end{array}$ & $\begin{array}{c}\text { Polyarticular } \\
n=22\end{array}$ & $\begin{array}{c}\text { SJIA } \\
n=21\end{array}$ & $\begin{array}{l}\text { ERA } \\
n=6\end{array}$ & \\
\hline \multicolumn{2}{|c|}{$\begin{array}{c}\text { Total Number of Examined } \\
\text { Joints }\end{array}$} & 280 & 160 & 880 & 840 & 240 & \\
\hline \multirow{4}{*}{$\begin{array}{l}\text { Number } \\
\text { joints with } \\
\text { effusion }\end{array}$} & Range & $0-12$ & $4-37$ & $0-26$ & $0-40$ & $2-16$ & \multirow{4}{*}{0.017 * } \\
\hline & Median & 4 & 30 & 7.5 & 14 & 5.5 & \\
\hline & (IQR) & (3) & $(24.75)$ & $(12.5)$ & $(20.5)$ & (9.5) & \\
\hline & $n$, percent & $\begin{array}{c}30 \\
(10.7 \%)\end{array}$ & $\begin{array}{c}101 \\
(63.1 \%)\end{array}$ & $\begin{array}{c}191 \\
(21.7 \%)\end{array}$ & $\begin{array}{c}334 \\
(40 \%)\end{array}$ & $\begin{array}{c}41 \\
(17 \%)\end{array}$ & \\
\hline \multirow{4}{*}{$\begin{array}{l}\text { Number } \\
\text { joints with } \\
\text { synovial } \\
\text { thickening }\end{array}$} & Range & $1-12$ & $4-30$ & $0-21$ & $2-40$ & $2-16$ & \multirow{4}{*}{$0.025 *$} \\
\hline & Median & 4 & 22.5 & 7 & 16 & 4 & \\
\hline & (IQR) & (3) & $(23.25)$ & $(92.5)$ & (20.5) & (8) & \\
\hline & $n$, percent & $\begin{array}{c}32 \\
(11.4 \%) \\
\end{array}$ & $\begin{array}{c}79 \\
(49.4 \%) \\
\end{array}$ & $\begin{array}{c}181 \\
(20.6 \%) \\
\end{array}$ & $\begin{array}{c}345 \\
(41 \%)\end{array}$ & $\begin{array}{c}36 \\
(15 \%) \\
\end{array}$ & \\
\hline \multirow{4}{*}{$\begin{array}{c}\text { Number } \\
\text { joints with } \\
\text { tenosynovitis }\end{array}$} & Range & 0 & $0-3$ & $0-2$ & $0-2$ & $0-3$ & \multirow{4}{*}{0.070} \\
\hline & Median & 0 & 2 & 0 & 0 & 0.5 & \\
\hline & (IQR) & (0) & $(2.25)$ & $(1.25)$ & (2) & $(2.25)$ & \\
\hline & $n$, percent & 0 & $\begin{array}{c}7 \\
(4.4 \%)\end{array}$ & $\begin{array}{c}13 \\
(1.5 \%)\end{array}$ & $\begin{array}{c}12 \\
(1.4 \%)\end{array}$ & $\begin{array}{c}6 \\
(2.5 \%)\end{array}$ & \\
\hline \multirow{4}{*}{$\begin{array}{l}\text { Number } \\
\text { joints with } \\
\text { erosion }\end{array}$} & Range & 0 & 0 & $0-3$ & $0-5$ & $0-2$ & \multirow{4}{*}{0.382} \\
\hline & Median & 0 & 0 & 0 & 0 & 0 & \\
\hline & (IQR) & $(0)$ & (0) & $(0.25)$ & (1) & $(1.25)$ & \\
\hline & $n$, percent & 0 & 0 & $\begin{array}{c}7 \\
(0.8 \%)\end{array}$ & $14(1.7 \%)$ & $3(0.8 \%)$ & \\
\hline \multirow{4}{*}{$\begin{array}{l}\text { Number } \\
\text { joints with } \\
\text { active } \\
\text { synovitis }\end{array}$} & Range & $0-4$ & $0-30$ & 0-19 & $0-40$ & $0-8$ & \multirow{4}{*}{0.115} \\
\hline & Median & 1 & 17 & 2 & 6 & 5 & \\
\hline & (IQR) & (3) & (29) & (7.25) & (21.5) & (5) & \\
\hline & $n$, percent & $\begin{array}{c}10 \\
(3.6 \%)\end{array}$ & $\begin{array}{c}64 \\
(40 \%)\end{array}$ & $\begin{array}{c}85 \\
(9.7 \%)\end{array}$ & $\begin{array}{c}261 \\
(31 \%)\end{array}$ & $\begin{array}{c}26 \\
(10.8 \%)\end{array}$ & \\
\hline \multirow{4}{*}{$\begin{array}{l}\text { Number } \\
\text { joints with } \\
\text { US synovitis }\end{array}$} & Range & $1-12$ & $4-37$ & $0-26$ & $2-40$ & $2-16$ & \multirow{4}{*}{$0.015^{*}$} \\
\hline & Median & 4 & 30 & 7.5 & 17 & 6 & \\
\hline & (IQR) & (3) & $(24.25)$ & (11.5) & (20.5) & $(9.5)$ & \\
\hline & $n$, percent & $\begin{array}{c}32 \\
(11.4 \%)\end{array}$ & $\begin{array}{c}101 \\
(63.1 \%)\end{array}$ & $\begin{array}{c}205 \\
(23.3 \%)\end{array}$ & $\begin{array}{c}350 \\
(41.7 \%)\end{array}$ & $\begin{array}{c}43 \\
(17.9 \%)\end{array}$ & \\
\hline
\end{tabular}


Table 4. Cont.

\begin{tabular}{|c|c|c|c|c|c|c|c|}
\hline \multicolumn{2}{|c|}{ US Features } & \multicolumn{5}{|c|}{ Subtypes of JIA } & \multirow{3}{*}{$\begin{array}{c}\text { Kruskal- } \\
\text { Wallis Test, } p \\
\text { Value }\end{array}$} \\
\hline & & $\begin{array}{l}\text { Oligoarticular } \\
\quad n=7\end{array}$ & $\begin{array}{l}\text { Extended- } \\
\text { Oligo } \\
n=4\end{array}$ & $\begin{array}{l}\text { Polyarticular } \\
\quad n=22\end{array}$ & $\begin{array}{c}\text { SJIA } \\
n=21\end{array}$ & $\begin{array}{l}\text { ERA } \\
n=6\end{array}$ & \\
\hline \multicolumn{2}{|c|}{$\begin{array}{c}\text { Total Number of Examined } \\
\text { Joints }\end{array}$} & 280 & 160 & 880 & 840 & 240 & \\
\hline \multirow{4}{*}{$\begin{array}{l}\text { Number } \\
\text { joints with } \\
\text { subclinical } \\
\text { synovitis }\end{array}$} & Range & $0-8$ & $3-20$ & $0-13$ & $0-15$ & $0-9$ & \multirow{4}{*}{0.017 * } \\
\hline & Median & 0 & 10 & 3.2 & 6 & 3 & \\
\hline & (IQR) & (3) & (12.75) & $(4.55)$ & $(6.3)$ & (8) & \\
\hline & $n$, percent & $\begin{array}{c}9 \\
(3.2 \%)\end{array}$ & $\begin{array}{c}58 \\
(36.25 \%)\end{array}$ & $\begin{array}{c}102 \\
(11.6 \%)\end{array}$ & $\begin{array}{c}126 \\
(15 \%)\end{array}$ & $\begin{array}{c}17 \\
(7.08 \%)\end{array}$ & \\
\hline \multirow{4}{*}{$\begin{array}{l}\text { Number of } \\
\text { joints with } \\
\text { low cartilage } \\
\text { thickness }\end{array}$} & Range & $0-8$ & $0-12$ & $0-12$ & $0-8$ & $0-12$ & \multirow{4}{*}{0.393} \\
\hline & median & 2 & 0 & 2 & 0 & 0 & \\
\hline & (IQR) & (8) & (9) & (4.5) & (2) & $(4.5)$ & \\
\hline & $n$, percent & $\begin{array}{c}26 \\
(9.3 \%)\end{array}$ & $\begin{array}{c}12 \\
(7.5 \%)\end{array}$ & $\begin{array}{c}66 \\
(7.5 \%)\end{array}$ & $\begin{array}{c}30 \\
(3.6 \%)\end{array}$ & $\begin{array}{c}14 \\
(5.8 \%)\end{array}$ & \\
\hline \multirow{3}{*}{$\begin{array}{l}\text { Number of } \\
\text { enthesitis }\end{array}$} & Range & $0-2$ & 0 & 0 & 0 & $0-6$ & \multirow{3}{*}{$<0.001 *$} \\
\hline & Median & 0 & 0 & 0 & 0 & 5 & \\
\hline & (IQR) & $(1.25)$ & $(0)$ & $(0)$ & (0) & (3) & \\
\hline \multirow{3}{*}{$\begin{array}{l}\text { Synovial } \\
\text { effusion score }\end{array}$} & Range & $0-15$ & $4-64$ & $0-45$ & $2-120$ & $2-16$ & \multirow{3}{*}{0.018 * } \\
\hline & Median & 4 & 55 & 12 & 18 & 7.5 & \\
\hline & (IQR) & (4) & $(47.5)$ & (16.25) & $(26.5)$ & (9.5) & \\
\hline \multirow{3}{*}{$\begin{array}{c}\text { Synovial } \\
\text { hypertrophy } \\
\text { score }\end{array}$} & Range & $1-13$ & $7-40$ & $0-30$ & $2-80$ & $2-16$ & \multirow{3}{*}{0.013 * } \\
\hline & Median & 4 & 24.5 & 8.5 & 20 & 6.5 & \\
\hline & (IQR) & (4) & $(27.5)$ & (11.5) & (27) & (9.5) & \\
\hline \multirow{3}{*}{$\begin{array}{l}\text { Active } \\
\text { synovitis } \\
\text { score }\end{array}$} & Range & $0-7$ & $0-30$ & $0-38$ & $0-80$ & $0-16$ & \multirow{3}{*}{0.157} \\
\hline & Median & 1 & 19 & 2.5 & 8 & 5 & \\
\hline & (IQR) & (4) & (28) & (8) & $(35.5)$ & (8.5) & \\
\hline \multirow{3}{*}{$\begin{array}{l}\text { Total US } \\
\text { severity score }\end{array}$} & Range & $2-35$ & $11-120$ & 0-96 & $4-280$ & $4-38$ & \multirow{3}{*}{$0.026^{*}$} \\
\hline & Median & 11 & 105.5 & 23.5 & 53 & 20 & \\
\hline & (IQR) & (9) & (89) & (34.5) & $(86.5)$ & (31) & \\
\hline
\end{tabular}

ERA: enthesitis-related arthritis, US: ultrasound, IQR: interquartile range. JADAS-10: juvenile arthritis disease activity score with 10 joints count, JSPADA: juvenile spondyloarthritis disease activity score, C-HAQ: childhood health assessment questionnaire, JAD: juvenile arthritis damage index. ${ }^{*}$ Statistically significant $p$-values.

Scores done by the MSUS for synovial effusion, hypertrophy, positive PD signals, and total US severity were all significantly correlated to different clinical parameters and disease assessment indices analyzed. Only joint erosion detected by US and the number of joints with low cartilage thickness were positively correlated to disease duration. Regarding the JSPADA, it was only correlated to the US activity score $(p=0.026)$ and total US severity score $(p=0.034)$. These scores were also significantly correlated to main laboratory investigations, except for hemoglobin, as summarized in Table 5. 
Table 5. Correlation between US features and laboratory findings.

\begin{tabular}{|c|c|c|c|c|c|c|c|c|}
\hline & \multicolumn{2}{|c|}{$\begin{array}{c}\text { Synovial } \\
\text { Effusion Score }\end{array}$} & \multicolumn{2}{|c|}{$\begin{array}{c}\text { Synovial } \\
\text { Hypertrophy Score }\end{array}$} & \multicolumn{2}{|c|}{$\begin{array}{c}\text { Active } \\
\text { Synovitis Score }\end{array}$} & \multicolumn{2}{|c|}{$\begin{array}{c}\text { Total } \\
\text { US Severity Score }\end{array}$} \\
\hline & $\mathbf{r}$ & $p$ & $\mathbf{r}$ & $p$ & $\mathbf{r}$ & $p$ & $\mathbf{r}$ & $p$ \\
\hline Hemoglobin(gm/dL) & -0.176 & 0.179 & -0.286 & 0.057 & -0.251 & 0.056 & -0.243 & 0.061 \\
\hline WBCs & 0.418 & $<0.001$ * & 0.366 & $<0.001$ * & 0.422 & $<0.001$ * & 0.408 & $<0.001$ * \\
\hline Platelets & 0.484 & $<0.001 *$ & 0.582 & $<0.001 *$ & 0.636 & $<0.001 *$ & 0.574 & $<0.001$ * \\
\hline ESR 1st hour & 0.492 & $<0.001 *$ & 0.614 & $<0.001 *$ & 0.680 & $<0.001$ * & 0.614 & $<0.001$ * \\
\hline CRP & 0.463 & $<0.001$ * & 0.501 & $<0.001$ * & 0.565 & $<0.001 *$ & 0.525 & $<0.001$ * \\
\hline
\end{tabular}

US: ultrasound, ESR: erythrocyte sedimentation rate, WBC: white blood cells, CRP: C-reactive protein. * Statistically significant $p$-values.

Furthermore, the US severity score was significantly higher in JIA patients who received steroids or combination therapy $(p=0.005)$ than in those not treated with them. Tocilizumab-treated patients showed a lower US score than non-tocilizumab-treated patients $(p<0.001)$.

The ROC curve was operated to assess the diagnostic utility of the active joint count and US active synovitis score in disease activity prediction in all JIA patients as illustrated in Table 6. In the oligoarticular subtype, the area under the curve (AUC) for the US activity score was 41.7 and for the active joint count was 62.5 . The US activity score cutoff of 7 had a sensitivity of $33.33 \%$, a specificity of $100 \%$, and an accuracy of $71.43 \%$ for disease activity detection. The active joint count value of 1 had a sensitivity of $100 \%$, a specificity of $25 \%$, and an accuracy of $57.14 \%$ for disease activity discrimination.

Table 6. The diagnostic utility of active joint count and US active synovitis score in prediction of disease activity in all JIA patients.

\begin{tabular}{cccc}
\hline & & US Activity Score & AJC \\
\hline Optimal Cut-off point & Value & 3 & 4 \\
\hline AUC & Value & 85.9 & 89.7 \\
\hline Sensitivity \% & $95 \%$ CI & $73.2-95.6$ & $81.2-98.1$ \\
\hline Specificity \% & Value & 85.7 & 78.57 \\
\hline & $95 \%$ CI & $67.33-95.97$ & $59.05-91.70$ \\
\hline PPV \% & Value & 78.75 & 90.62 \\
\hline NPV $\%$ & $95 \%$ CI & $49.99-86.88$ & $74.98-98.02$ \\
\hline & Value & 70.59 & 88.00 \\
\hline Accuracy \% & $95 \%$ CI & $58.41-80.40$ & $71.05-95.63$ \\
\hline & Value & 84.62 & $70.22-90.83$ \\
\hline & $95 \%$ CI & $68.31-93.35$ & 85.00 \\
\hline & Value & 83.67 & $73.43-92.90$ \\
\hline
\end{tabular}

US: ultrasound, AJC: active joint count, AUC: area under curve, CI: confidence interval, PPV: positive predictive value, NPV: negative predictive value.

For polyarticular JIA patients, the AUC for the US activity score and the active joint count was 70.5 and 79.9 , respectively. The sensitivity of the US activity score cutoff value equivalent to 3 reached $75 \%$. Meanwhile, this cutoff value had a specificity of $64.29 \%$ and an accuracy of $68.18 \%$ for disease activity prediction. Furthermore, the active joint count cutoff of 4 had a sensitivity of $62.50 \%$, a specificity of $92.86 \%$, and an accuracy of $81.82 \%$ for disease activity discrimination. 
In children with SJIA, the AUC for the US activity score and the active joint count was 99.5 and 98.2, respectively. The cutoff value of the US activity score of 8 had a sensitivity of $100 \%$, a specificity of $90 \%$, and an accuracy of $95.24 \%$ for disease activity discernment. For disease activity prediction, the active joint count cutoff of 4 had a sensitivity of $100 \%$, a specificity of $80 \%$, and an accuracy of $90.48 \%$.

In ERA, the AUC for the US activity score was 100 and for the active joint count was 87.5. The US activity score cutoff of 6 provided a sensitivity of $75 \%$, a specificity of $100 \%$, and an accuracy of $83.33 \%$ for disease activity detection. The cutoff value of the active joint count at 4 had a sensitivity of $75 \%$, a specificity of $100 \%$, and an accuracy of $83.33 \%$ for disease activity estimation.

\section{Discussion}

JIA is an umbrella term that includes a group of clinically different subtypes characterized by joint inflammation and destruction, which lead to severe disability and poor functional outcomes [14].

By US examination of JIA patients, the most frequent features detected were synovial effusion in 697 of total 2400 examined joints (29\%); synovial thickening in $673(28 \%)$, and positive PD signals in 446 (18.6\%). In harmony with these findings, Lotfy et al., 2018 [15], were concerned with the value of PD for the disease activity detection in 40 JIA patients and reported that the main US findings were synovial thickening and positive PD signals. The least detected feature was erosion in 24 joints (1\%), and this runs with Collado et al., 2014 [16] (two patients only), and Ventura-Ríos et al., 2018 [17] (12/60 MCP joints). This percentage was lower than Darwish et al.'s 2016 [18] observation (9.75\% of examined joints had erosion).

This may be attributed to the longer disease duration in their studies or the older age of included children. Unlike RA, subchondral bone erosions are not frequently observed in JIA, and the interpretation of bone erosions is challenging because some irregularities of ossified bones can be misinterpreted as cortical erosions [19].

The number of joints with low cartilage thickness was $146(0.6 \%)$ in our JIA subjects; such joint space narrowing as an early destructive change in JIA was reported in various papers $[15,20]$. Enthesitis was detected in 28 entheses sites $(4.6 \%)$, and the most common sites of enthesitis were the Achilles tendon followed by distal patellar tendon and plantar fascia. Similarly, the US confirmed enthesitis at the Achilles tendon insertion in the majority of cases examined by Kiris et al., 2006 [21]. Enthesitis is not exclusive to ERA, as it was found in two oligoarticular cases as reported by Weiss et al., 2012 [8].

In accordance with our observation, Magni-Manzoni et al., 2009 [22], carried out US examination of 32 JIA patients, and they observed that subclinical synovitis was detected in $10 \%$ of examined joints. Moreover, the US similarly resulted in re-classification of four cases from oligo to polyarthritis.

A previous study [15] confirmed our finding: US abnormalities were most frequently found in knees followed by wrists and MCP joints. In concordance with a prior study [23], there was a significant difference among JIA subtypes as regards different US features attributed to the higher US scores in SJIA cases. Scores done by the US for synovial effusion, hypertrophy, positive PD signals, and the total US severity score were all significantly correlated to most of disease assessment indices. In the same way, Borzani et al., 2015 [24] stated that US scores were correlated with the number of swollen joints, the active joint count, and the clinical JADAS-10.

Our finding concerning the accuracy of the PDUS in JIA disease activity detection is consistent with the work reported by Nielsen and his colleagues [25]. However, Beck et al., 2017 [26], reported a lower sensitivity of the PD (32.5\%) in disease activity detection. On comparison between the US and clinical examination, both had similar accuracy in our study and nearby sensitivity and specificity, while Darwish et al., 2016 [18] concluded that the sensitivity of clinical examination was $34.5 \%$ compared to the US (45.7\%), and the specificity was $100 \%$ for both. 
This study provides, for the first time, the sensitivity, specificity, PPV, NPV, and accuracy of PDUS in different JIA subtypes, which will be helpful for further research work about the diagnostic utility of US in JIA subtypes. Eventually, no single assessment tool can be sufficiently sensitive or specific, especially in a heterogeneous disease such as JIA, which can follow different patterns and respond variably to treatment strategies [27]. Thus, a multimodal panel of clinical, biomarker, and MSUS is fundamental for accurate assessment.

\section{Conclusions}

MSUS is a highly sensitive method for detecting synovitis, tenosynovitis, and erosive bone disease. It is a useful tool in routine clinical practice and helps to make proper therapeutic decisions. There were significant differences between JIA subtypes regarding US features with skewing of US scores to SJIA.

Author Contributions: Conceptualization, D.M.M. and A.S.E.-B.; methodology, D.M.M., A.M.A. and A.S.E.-B.; software, D.M.M. and A.S.E.-B.; validation, D.M.M., A.M.A. and A.S.E.-B.; formal analysis, D.M.M., A.M.A. and A.S.E.-B.; investigation, D.M.M. and A.M.A.; resources, D.M.M. and A.M.A.; data curation, D.M.M., A.M.A. and A.S.E.-B.; writing-original draft preparation, D.M.M.; writing-review and editing, D.M.M., A.M.A. and A.S.E.-B.; visualization, D.M.M., A.M.A. and A.S.E.-B.; supervision, A.M.A. and A.S.E.-B.; project administration, D.M.M. and A.S.E.-B. All authors have read and agreed to the published version of the manuscript.

Funding: No specific funding was received from any bodies in the public, commercial or not-for-profit sectors to carry out the work described in this article.

Institutional Review Board Statement: The study was conducted after the approval of institutional research board (IRB) of the Faculty of Medicine, Mansoura University, Egypt (approval number: M 1900483).

Informed Consent Statement: Patient whose parents sign a consent to be included in the study.

Data Availability Statement: All data are available on this manuscript.

Conflicts of Interest: The authors declare no conflict of interest.

\section{References}

1. Huang, J.L. New advances in juvenile idiopathic arthritis. Chang. Gung Med. J. 2012, 35, 1-14. [CrossRef] [PubMed]

2. Petty, R.E.; Southwood, T.R.; Manners, P.; Baum, J.; Glass, D.N.; Goldenberg, J.; He, X.; Maldonado-Cocco, J.; Orozco-Alcala, J.; Prieur, A.-M.; et al. International League of Associations for Rheumatology classification of juvenile idiopathic arthritis. J. Rheumatol. 2004, 31, 390-392. [PubMed]

3. Lovell, D.J.; Ruperto, N.; Goodman, S.; Reiff, A.; Jung, L.; Jarosova, K.; Němcova, D.; Mouy, R.; Sandborg, C.; Bohnsack, J.; et al. Adalimumab with or without methotrexate in juvenile rheumatoid arthritis. N. Engl. J. Med. 2008, 359, 810-820. [CrossRef] [PubMed]

4. Scheel, A.K.; Hermann, K.G.; Ohrndorf, S.; Werner, C.; Schirmer, C.; Detert, J.; Bollow, M.; Hamm, B.; Müller, G.A.; Burmester, G.R.; et al. Prospective 7-year follow-up imaging study comparing radiography, ultrasonography and magnetic resonance imaging in rheumatoid arthritis finger joints. Ann. Rheum. Dis. 2006, 65, 595-600. [CrossRef] [PubMed]

5. Breton, S.; Jousse-Joulin, S.; Finel, E.; Marhadour, T.; Colin, D.; de Parscau, L.; Devauchelle-Pensec, V. Imaging approaches for evaluating peripheral joint abnormalities in juvenile idiopathic arthritis. Semin. Arthritis Rheum. 2012, 41, 698-711. [CrossRef] [PubMed]

6. Lanni, S.; Wood, M.; Ravelli, A.; Magni Manzoni, S.; Emery, P.; Wakefield, R.J. Towards a role of ultrasound in children with juvenile idiopathic arthritis. Rheumatology 2013, 52, 413-420. [CrossRef]

7. Consolaro, A.; Bracciolini, G.; Ruperto, N. Remission, minimal disease activity and acceptable symptom state in juvenile idiopathic arthritis. Arthritis Rheumatol. 2012, 64, 2366-2374. [CrossRef]

8. Weiss, P.F.; Beukelman, T.; Schanberg, L.E.; Kimura, Y.; Colbert, R.A. Enthesitis-related arthritis is associated with higher pain intensity and poorer health status in comparison with other categories of juvenile idiopathic arthritis: The Childhood Arthritis and Rheumatology Research Alliance Registry. J. Rheumatol. 2012, 39, 2341-2351. [CrossRef]

9. Viola, S.; Felici, E.; Magni-Manzoni, S.; Pistorio, A.; Buoncompagni, A.; Ruperto, N.; Rossi, F.; Bartoli, M.; Martini, A.; Ravelli, A.; et al. Development and validation of a clinical index for assessment of long-term damage in juvenile idiopathic arthritis. Arthritis Rheumatol. 2005, 52, 2092-2102. [CrossRef]

10. Tennant, A.; Kearns, S.; Turner, F.; Wyatt, S.; Haigh, R.; Chamberlain, M.A. Measuring the function of children with juvenile arthritis. Rheumatology 2001, 40, 1274-1278. [CrossRef] 
11. Collado, P.; Vojinovic, J.; Nieto, J.C.; Windschall, D.; Magni-Manzoni, S.; Bruyn, G.A.; Iagnocco, A.; D’Agostino, M.A.; Naredo, E.; on behalf of the Omeract Ultral Pediatric Group. Toward standardized musculoskeletal ultrasound in pediatric rheumatology: Normal age-related ultrasound findings. Arthritis Care Res. 2016, 68, 348-356. [CrossRef] [PubMed]

12. Roth, J.; Ravagnani, V.; Backhaus, M.; Balint, P.; Bruns, A.; Bruyn, G.A.; Collado, P.; De La Cruz, L.; Guillaume-Czitrom, S.; Herlin, T.; et al. Preliminary definitions for the Sonographic features of Synovitis in children. Arthritis Care Res. 2017, 69, 1217-1223. [CrossRef] [PubMed]

13. Vojinovic, J.; Magni-Manzoni, S.; Collado, P.; Windschall, D.; Ravagnani, V.; Hernandez-Diaz, C.; Gonzales, J.C.N.; Malattia, C.; Tzaribachev, N.; Susic, G.; et al. SAT0636 Ultrasonography definitions for synovitis grading in children: The omeract pediatric ultrasound task force. Ann. Rheum. Dis. 2017, 76, 1015.

14. Prakken, B.; Albani, S.; Martini, A. Juvenile idiopathic arthritis. Lancet 2011, 377, 2138-2149. [CrossRef]

15. Lotfy, H.; Seif El Dienb, H.; El Minawia, N.; Abdel Aziz, H. The role of Doppler Ultrasonography in evaluating disease activity in a group of juvenile idiopathic arthritis patients. Egypt. J. Radiol. Nucl. Med. 2018, 49, 1036-1042. [CrossRef]

16. Collado, P.; Gamir, M.L.; López-Robledillo, J.C.; Merino, R.; Modesto, C.; Monteagudo, I. Detection of synovitis by ultrasonography in clinically inactive juvenile idiopathic arthritis on and off medication. Clin. Exp. 2014, 32, 597-603.

17. Ventura-Ríos, L.; Faugier, E.; Barzola, L.; De La Cruz-Becerra, L.B.; Sánchez-Bringas, G.; García, A.R.; Maldonado, R.; Roth, J.; Hernández-Día, C.Z. Reliability of ultrasonography to detect inflammatory lesions and structural damage in juvenile idiopathic arthritis. Pediatr. Rheumatol. 2018, 16, 58. [CrossRef]

18. Darwish, A.; Ismaela, F.; Ell-Labana, A.; Hamed, A.; Kader, M.A.; Osman, A. Implementation of musculoskeletal ultrasonography in detection of early juvenile idiopathic arthritis. Eur. J. Radiol. 2016, 3, 264-271. [CrossRef]

19. Laurell, L.; Court-Payen, M.; Nielsen, S.; Zak, M.; Thomsen, C.; Miguel-Pérez, M.; Fasth, A. Ultrasonography and color Doppler of proximal gluteal enthesitis in juvenile idiopathic arthritis: A descriptive study. Pediatr. Rheumatol. Online J. 2011, 9, 22. [CrossRef]

20. Pradsgaard, D.Ø.; Spannow, A.H.; Heuck, C.; Herlin, T. Decreased cartilage thickness in juvenile idiopathic arthritis assessed by ultrasonography. J. Rheumatol. 2013, 40, 1596-1603. [CrossRef]

21. Kiris, A.; Kaya, A.; Ozgocmen, S.; Kocakoc, E. Assessment of enthesitis in ankylosing spondylitis by power Doppler ultrasonography. Skelet. Radiol. 2006, 35, 522-528. [CrossRef] [PubMed]

22. Magni-Manzoni, S.; Epis, O.; Ravelli, A. Comparison of clinical versus ultrasound-determined synovitis in juvenile idiopathic arthritis. Arthritis Rheumatol. 2009, 61, 1497-1504. [CrossRef] [PubMed]

23. El-Banna, H.; Nada, D.; Hussein, M.; Hablas, S.; Darwish, N.; Abu-Zaid, M.; Gadou, S.E. Role of musculoskeletal ultrasonography in the detection of subclinical synovitis in oligo and polyarticular juvenile idiopathic arthritis children. Egypt. Rheumatol. 2019, 41, 151-155. [CrossRef]

24. Borzani, I.; Di Landro, G.; Ravelli, A.; Corona, F.; Filocamo, G. SAT0506 Towards the Development of an Ultrasound Composite Disease Activity Score for Juvenile Idiopathic Arthritis. Ann. Rheum. Dis. 2015, 74, 843. [CrossRef]

25. Nielsen, H.E.; Strandberg, C.; Andersen, S.; Kinnander, C.; Erichsen, G. Ultrasonographic examination in juvenile idiopathic arthritis is better than clinical examination for identification of intraarticular disease. Dan. Med. J. 2013, 60, A4669. [PubMed]

26. Beck, M.C.; Glimm, A.M.; Ohrndorf, S.; Minden, K.; Trauzeddel, R.; Werner, S.; Horneff, G.; Backhaus, M.; Burmester, G.R.; Kallinich, T.; et al. Fluorescence optical imaging in pediatric patients with inflammatory and non-inflammatory joint diseases: A comparative study with ultrasonography. Arthritis Res. Ther. 2017, 19, 233. [CrossRef] [PubMed]

27. Vojinovic, J. Toward a Multibiomarker Panel to Optimize Outcome and Predict Respons. J. Rheumatol. 2018, 45, 451-453. [CrossRef] 